\title{
Evaluation and correction of aberrations in an optical correlator by phase-shifting interferometry
}

\author{
Claudio Iemmi \\ Departamento de Física, Facultad de Ciencias Exactas y Naturales, Universidad de Buenos Aires, (1428) Buenos Aires, Argentina
}

\author{
Alfonso Moreno, Josep Nicolás, and Juan Campos \\ Departamento de Física, Universidad Autónoma de Barcelona, 08193 Bellaterra, Spain
}

Received December 3, 2002

\begin{abstract}
We propose a new method for evaluating and correcting aberrations in a Vander Lugt correlator. The technique is achieved with liquid-crystal displays of the correlator and allows the task to be performed in situ. We present the theory on which the method is based and the experimental results that we obtained by applying it in a convergent correlator. (C) 2003 Optical Society of America

OCIS codes: $100.4550,120.3180,120.5050$.
\end{abstract}

During the past decade, twisted nematic liquid-crystal displays (LCDs) have been widely used in pattern recognition, ${ }^{1-3}$ mainly because of their capacity to display images at video rates and to act as programmable spatial light modulators. In Vander Lugt-type correlators, both the input scene and the filter can be implemented onto these elements. Usually the scene is displayed as an amplitude distribution; however, there are some proposals in which the input is displayed as a phase distribution ${ }^{4,5}$ to improve discrimination capability and light efficiency. The filter usually works as a phase modulator. It should be pointed out that, in general, LCDs produce coupled amplitude and phase modulation when they are used, as usual, between linear polarizers. Recently a physical model for twisted nematic LCDs was presented that predicts the configuration of the polarizing elements that will produce the desired modulation (pure phase or pure amplitude) through the use of elliptically polarized light. ${ }^{6}$

Misalignments and aberrations seriously affect the performance of optical correlators. Several authors have studied the incidence of misalignments in correlation results, ${ }^{7,8}$ and different techniques to correct this problem have been proposed. In Ref. 9 an automated procedure based on an iterative process to correct the transversal misalignment of a scene or a filter was reported. In Ref. 3 a series of frequencyanalysis-based tests was described with which the elements of a LCD-based convergent correlator can be aligned accurately both at building time and at working time. An analysis of the influence of wave aberrations in convergent correlators is presented in Ref. 10; the authors showed that shift invariance is affected by the aberrations introduced by the optical system between the input scene and the filter. These aberrations may cause the Fourier transforms of different zones of the input scene to have different centers in the filter plane; then the filter cannot be aligned for the entire scene. The aberrations introduced after the Fourier plane affect image formation at the correlation plane in a global way, i.e., they are not critical for shift invariance. The evaluation and correction of the aberrations of the first part of the system seem to be more critical. Wave aberrations could be introduced by lenses or by any of the elements (e.g., spatial light modulators, polarizers, wave plates) that constitute the system.

Usually configurations with which amplitude and phase modulation can be obtained from a LCD are highly sensitive to input and output polarization element angles; consequently, characterization of spatial light modulators in situ, i.e., in the correlator setup, is desirable. ${ }^{11}$ Moreover, aberrations introduced by modulators and the various elements depend strongly on the orientation of all elements; thus to evaluate these aberrations it is necessary to develop a method that allows this task to be performed in situ.

In this Letter we propose a new method in which the correlator acts as an interferometer and phase extraction is performed by the phase-shift interferometry technique. In Fig. 1 an illustration of a convergent correlator is shown. When it is used in a pattern-recognition process the input scene is displayed on LCD1 and the filter on LCD2. LCD2 is placed in the conjugate plane of the source, s, i.e., where the Fourier transform of LCD1 is obtained. Both LCDs are working to give phase-only modulation. Our goal is to evaluate and to correct the aberrations introduced by all the elements placed before the Fourier plane because, as we have explained, they are the most critical part of the performance

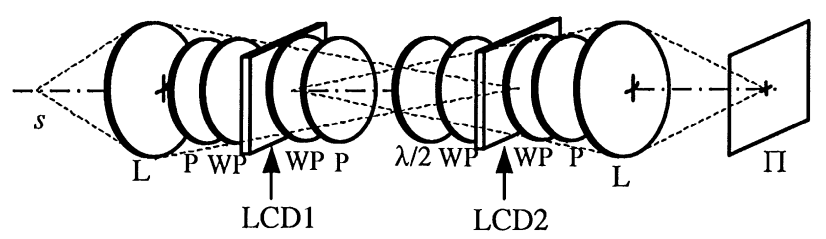

Fig. 1. Schematic of a convergent optical correlator; Ls, convergent lenses; WPs, wave plates; Ps, polarizers. The scene and the filter are displayed on spatial light modulators LCD1 and LCD2, respectively. $\Pi$ represents the final plane. 
of the optical correlator. Modulation of the LCD depends on the incidence angle. Our correlator is very long (more than $2 \mathrm{~m}$ ), and the influence of the incidence angle can be neglected. However, for compact correlators one should use additional lenses as described in Ref. 12 to obtain normal incidence on the LCDs.

We describe our method: When no image is displayed in LCD1 and in the absence of aberrations, a bright central spot corresponding to the Fourier transform of the ideal entrance pupil will be obtained on LCD2. If some aberrations are introduced by the elements placed in front of the transformed plane, that bright central spot will be deformed. The image of this phaseaberrated wave front can become a gray-level image in the final plane ( $\Pi$, Fig. 1) by application of phase retardation in the center of the Fourier transform on LCD2, similarly to what Zernike proposed for the phase-contrast microscope. ${ }^{13}$ The perturbation that originates at the central point acts as a reference wave that interferes with the aberrated wave front. The basic idea of the phase-shift interferometry technique is to change the phase of the reference beam in a known manner and then calculate the phase of the wave front from a set of intensities measured at the interferogram plane. ${ }^{14}$ In our case the phase shifts are introduced by the central pixel of LCD2, which, as we mentioned above, is working as a phase modulator. Mathematically, the filter transfer function that we introduced into LCD2 is

$$
H(u)=1+\delta(u)\left[\exp \left(i \frac{2 \pi n}{N}\right)-1\right]
$$

where $\delta(u)$ is the Kronecker function, which takes the value 1 when $u=0$ and the value 0 elsewhere, and $2 \pi n / N$ is the plane shift introduced in each of the $N$ steps that we applied to obtain the unknown phase. The intensity transmission of this filter is uniform, but the phase of the pixel at the origin is shifted with respect to the other pixels by $2 \pi n / N$ rad. The amplitude at the final plane is given by the expression

$$
\begin{aligned}
A_{n}(x) & =f(x) \exp [i \Psi(x)] * h(x) \\
& =f(x) \exp [i \Psi(x)]+\left[\exp \left(i \frac{2 \pi n}{N}\right)-1\right] K,
\end{aligned}
$$

where $f(x) \exp [i \Psi(x)]$ is the unknown complex amplitude of the aberrated wave front, $h(x)$ is the filter impulse response, $*$ denotes convolution, and complex constant $K=|K| \exp (i \mu)$ is the mean value of $f(x) \exp [i \Psi(x)]$.

In the final plane the square module of amplitude $A_{n}(x)$ is detected. This intensity is the interference between the problem function and a reference wave that depends on $n$. To obtain phase information $\psi(x)$ we measure several interference patterns $\left|A_{n}\right|^{2}$, with $n=0, \ldots, N-1$. Following the synchronous detection of interference fringes ${ }^{15}$ the measured intensities $\left|A_{n}\right|^{2}$ are multiplied by $\cos (2 \pi n / N)$ and $\sin (2 \pi n / N)$ according to the following expressions:

$$
\begin{aligned}
C & =\sum_{n=0}^{N-1}\left(A_{n} A_{n}{ }^{*}\right) \cos \left(\frac{2 \pi n}{N}\right), \\
S & =\sum_{n=0}^{N-1}\left(A_{n} A_{n}{ }^{*}\right) \sin \left(\frac{2 \pi n}{N}\right),
\end{aligned}
$$

respectively. Taking advantage of the orthogonality properties of the sinusoidal functions and replacing in Eq. (3) the expression for $A_{n}$ given in Eq. (2), we find that

$$
\begin{aligned}
& C=-N|K|^{2}+N|K| f(x) \cos [\Psi(x)+\mu], \\
& S=N|K| f(x) \sin [\Psi(x)+\mu] .
\end{aligned}
$$

Finally, the unknown phase $\Psi(x)$ can be obtained as

$$
\Psi(x)=\tan ^{-1}\left(S / C-C_{0}\right)-\mu,
$$

where $\mu$ is a constant phase and $C_{0}=-N|K|^{2}$. We obtain the value of $C_{0}$ by evaluating Eqs. (4) at those points at which $f(x)=0$. In the image plane this region corresponds to the zone that lies outside the circular diaphragm and upon which no light impinges when the phase shift is zero [see Fig. 2(a)]. When a phase shift is introduced into the filter, the light diffracted by the central pixel produces an intensity distribution that is almost constant over the region. This is so because the size of the pinhole image (image of the light source) at the Fourier plane is larger than 1 pixel. For example, an image corresponding to expression $C$ is shown in Fig. 2(b). It is noticeable that the intensity distribution outside the circle is practically uniform; then, by taking an average of the pixel values that belong to that zone, one can obtain the $C_{0}$ value to a good approximation.

In our experiments we used the violet line $(\lambda=458 \mathrm{~nm})$ of an Ar laser as the light source. The LCDs that we used were Sony LCX012BL with VGA resolution $(640 \times 480$ pixels $)$ extracted from a Sony video projector, and we obtained the phase modulation by following the procedure described in Ref. 6.

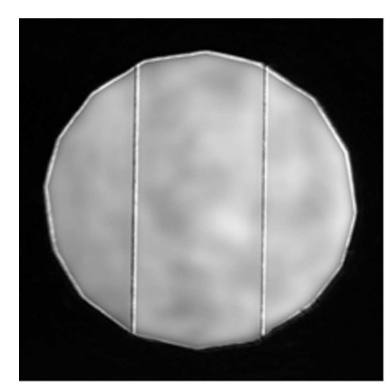

(a)

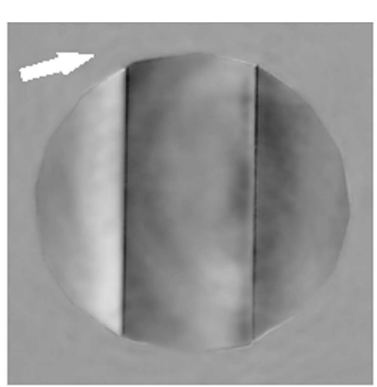

(b)
Fig. 2. (a) Image of the final plane when no phase shift is introduced into the filter. In this case light does not impinge in the zone placed outside the circular diaphragm. (b) Image corresponding to expression $C$. One can evaluate the first term, $C_{0}$, by taking an average of pixel values over that external zone (white arrow). 


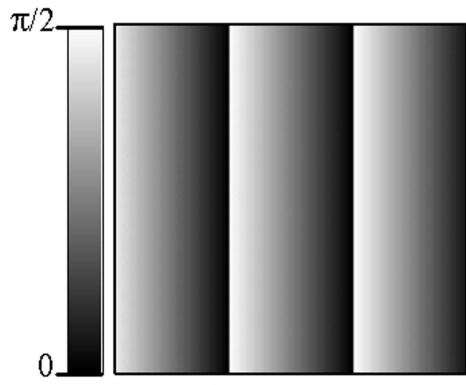

(a)

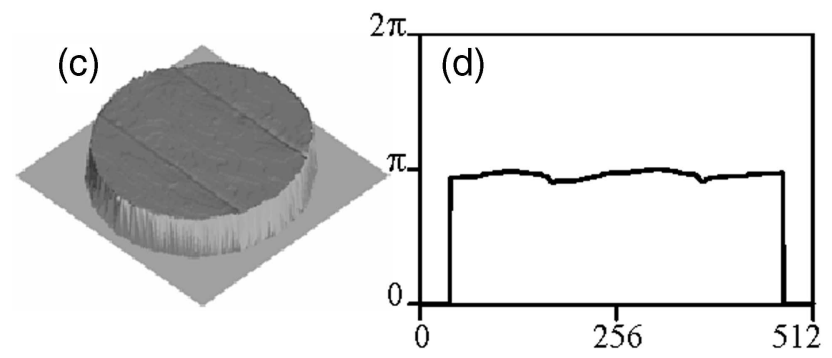

Fig. 3. (a) Theoretical phase distribution, (b) experimental phase extraction, (c) result of the phase extraction obtained when the theoretical phase and the experimental phase were subtracted and displayed in LCD1, (d) line profile of (c).

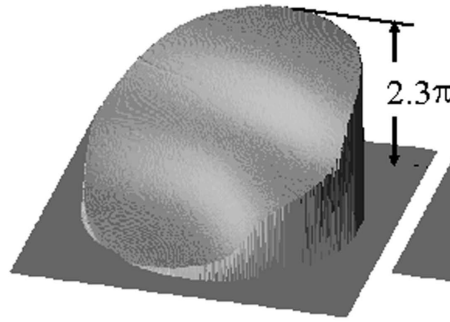

(a)

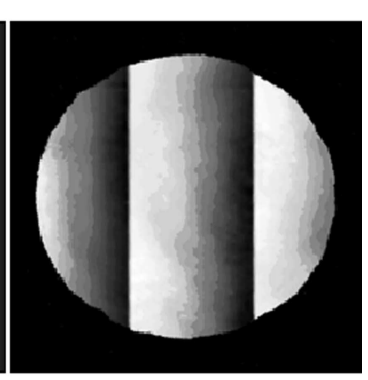

(b)
Fig. 4. (a) Measure of the phase distribution introduced by a piece of glass, (b) measure of the phase distribution after correction.

First, to test the method we displayed a known phase distribution on LCD1. It was a saw-toothed profile from 0 to $\pi / 2$. In Fig. 3(a) a gray-level representation of this theoretical phase distribution is drawn. Phase extraction was performed over a 20-mmdiameter circular area, and the values obtained experimentally are shown in Fig. 3(b). The theoretical distribution was subtracted from the experimental one, and the result was displayed again in LCD1. A new phase extraction was performed, and the result obtained is shown in a three-dimensional representation in Fig. 3(c). It is noticeable from the profile represented in Fig. 3(d) that the original phase was canceled successfully with an error smaller than $\lambda / 20$.

In a second test we placed between LCD1 and LCD2 a piece of glass that introduced unknown aberrations into the system. By following the proposed method we evaluated the phase distortion that is shown in
Fig. 4(a). The range of the phase distortion is $\sim 2.3 \pi$. By displaying in LCD1 the conjugate of this phase, and by again evaluating the wave front, we obtained the corrected phase shown in Fig. 4(b). In this case the phase range was no bigger than $0.13 \pi$. Let us say that we have corrected the wave aberration of the system.

It should be pointed out that the aberrations introduced by the set of elements that constitute the filter (LCD2, wave plates, and polarizers) have negligible influence on the phase extraction process. This is so because the light distributed at the Fourier plane is focused to a small spot.

Summarizing, we have demonstrated that the proposed method allows us to evaluate and correct in situ an arbitrary phase distribution caused by aberrations introduced by the various elements that constitute an optical correlator. The phase extraction is performed by application of the phase-shift interferometry technique and use of the correlator itself as an interferometer without employing any other element.

J. Nicolás thanks the Comissió d'Investigació de la Universitat Autònoma de Barcelona for a grant. This research was partially financed by the Ministerio de Ciencia y Tecnología (Spain) under project BFM2000-0036-C02-01. C. Iemmi acknowledges the support of the Consejo Nacional de Investigaciones Cientificas y Tecnicas and the Universidad de Buenos Aires (Argentina) and the Secretaría de Estado de Educación y Universidades (Spain). J. Campos's e-mail address is juan.campos@uab.es.

\section{References}

1. H. K. Liu, J. A. Davis, and R. A. Lilly, Opt. Lett. 10, 635 (1985).

2. C. Soutar, Z. Q. Wang, C. M. Cartwright, and W. A. Gillespie, J. Mod. Opt. 39, 761 (1992).

3. J. Nicolás, J. Campos, C. Iemmi, I. Moreno, and M. J. Yzuel, Appl. Opt. 41, 1505 (2002).

4. R. R. Kallman and D. H. Goldstein, Opt. Eng. 33, 1806 (1994).

5. K. Styczynski, J. Campos, M. J. Yzuel, and K. Chalasinska Macukow, Opt. Commun. 118, 193 (1995).

6. A. Marquez, C. Iemmi, I. Moreno, J. A. Davis, J. Campos, and M. J. Yzuel, Opt. Eng. 40, 2558 (2001).

7. A. Vander Lugt, Appl. Opt. 6, 1221 (1967).

8. L. Cai, Y. Jin, S. Zhou, P. Yeh, N. Marzwell, and H. Liu, Opt. Eng. 35, 415 (1996).

9. M. Montes Usategui, S. E. Monroe, and R. D. Juday, Opt. Eng. 36, 1782 (1997).

10. J. Pérez Tudela, M. Montes Usategui, I. Juvells, and S. Vallmitjana, Opt. Commun. 184, 345 (2000).

11. O. López Coronado, I. Moreno, J. Campos, and M. J. Yzuel, J. Mod. Opt. 45, 2461 (1998).

12. H. J. Caulfield, M. P. Schamschula, and L. Zhang, Opt. Lett. 20, 1803 (1995).

13. F. Zernike, Z. Tech. Phys. 16, 454 (1935).

14. K. Creath, in Progress in Optics, E. Wolf, ed., (Elsevier, Amsterdam, 1988), Vol. XXVI, pp. 350-393.

15. D. Malacara, Optical Shop Testing (Wiley, New York, 1978). 